\title{
On the vertical and horizontal transverse oscillations of curved coronal loops
}

\author{
M. S. Ruderman \\ Department of Applied Mathematics, University of Sheffield, Hicks Building, Hounsfield Road, Sheffield S3 7RH, UK \\ e-mail: M.S.Ruderman@sheffield.ac.uk
}

Received 8 June 2009/ Accepted 10 August 2009

\begin{abstract}
Kink oscillations of curved coronal loops with the density varying along the loop are studied in the thin tube approximation. The equilibrium magnetic field is assumed to be potential, and the field potential and flux function are used as curvilinear coordinates. It is also assumed that the loop expansion is weak, and the solution to the problem is looked for in the form of power series with respect to the tube expansion parameter $\lambda \ll 1$. The main result of the study is that the eigenfrequencies of the vertical and horizontal tube oscillations are, in general, different, their difference being proportional to $\lambda$. As an example a simple equilibrium with the magnetic field magnitude exponentially decaying with the height is considered. The implication of the obtained results for the interpretation of observational data is discussed.
\end{abstract}

Key words. magnetohydrodynamics (MHD) - waves - Sun: oscillations - Sun: corona

\section{Introduction}

Since transverse oscillations of coronal loops were first observed by TRACE (Aschwanden et al. 1999; Nakariakov et al. 1999), they received ample attention from both observers and theorists. The majority of observed transverse oscillations of coronal loops were polarised in the horizontal direction. However, Wang \& Solanki (2004) and Wang et al. (2008) reported the observations by TRACE of the vertically polarised transverse oscillations of coronal loops. These observations raise a question: what is the difference between properties of the horizontally and vertically polarised oscillations? In particular, do they have the same frequencies, or their frequencies are different?

In first theoretical studies of transverse oscillations of coronal loops the simplest model of a coronal loop was used. In this model a coronal loop is considered as a straight homogeneous magnetic tube with the footpoints frozen in the dense photospheric plasma. Since this magnetic plasma configuration is axisymmetric, the loop oscillations can be polarised in any direction, and the oscillation frequency is independent of the polarisation direction. Then more complicated models taking such effects as the density variation along and across a loop, the loop expansion and curvature were developed (see review paper by Ruderman \& Erdélyi 2009, and references therein). In particular, Van Doorsselaere et al. (2004) considered transverse oscillations of curved coronal loops. They assumed that the loop has the form of a half-circle and neglected the coronal density stratification. Using the toroidal coordinates they studied the transverse loop oscillations in the thin tube approximation. Their conclusion was that the eigenmode frequencies of the transverse loop oscillations remain unchanged up to first order in curvature. The curvature has more pronounced effect on the damping rate caused by resonant absorption in a thin boundary layer near the tube boundary. The correction to the damping rate due to the curvature is of the first order in curvature.
Later Terradas et al. (2006) studied the same problem as Van Doorsselaere et al. (2004), however taking the density stratification into account. Since Terradas et al. (2006) did not use the thin tube approximation but rather solved the exact linearised MHD equations, they managed to calculate corrections to the oscillation frequencies due to curvature. As a result, they found that the curvature removes the degeneration of eigenmodes of the transverse loop oscillations in a sense that now the eigenmodes can be polarised either in the horizontal or in the vertical direction only. However for thin coronal loops the difference between the eigenfrequencies of the two modes is very small.

Terradas et al. (2006) also studied the damping of transverse oscillations. In contrast to Van Doorsselaere et al. (2004) they did not assume that the inhomogeneous layer where the density varies in the radial direction is thin. Rather they allowed the density to vary in a layer with an arbitrary thickness. Terradas et al. (2006) confirmed the conclusion by Van Doorsselaere et al. (2004) that curvature has mode pronounced effect on the damping rate than on the eigenfrequencies. In addition, they found that, due to the density stratification, the oscillations become leaky and can be in resonance with the local Alfvén oscillations in the external plasmas. However the damping due to resonant absorption in the boundary layer near the tube boundary strongly dominates the damping due to leakage and resonance in the external plasma.

A very important assumption made by both Van Doorsselaere et al. (2004) and Terradas et al. (2006) was that the loop cross-section is circular and its radius does not very along the loop. These conditions are hardly satisfied in real coronal loops. The loop expansion leads to the variation of the loop cross-section area along the loop. In addition, the loop curvature can cause the variation of the cross-section shape. In this paper we study the effect of the variation of the loop cross-section on the transverse coronal loop oscillations using a simple model 


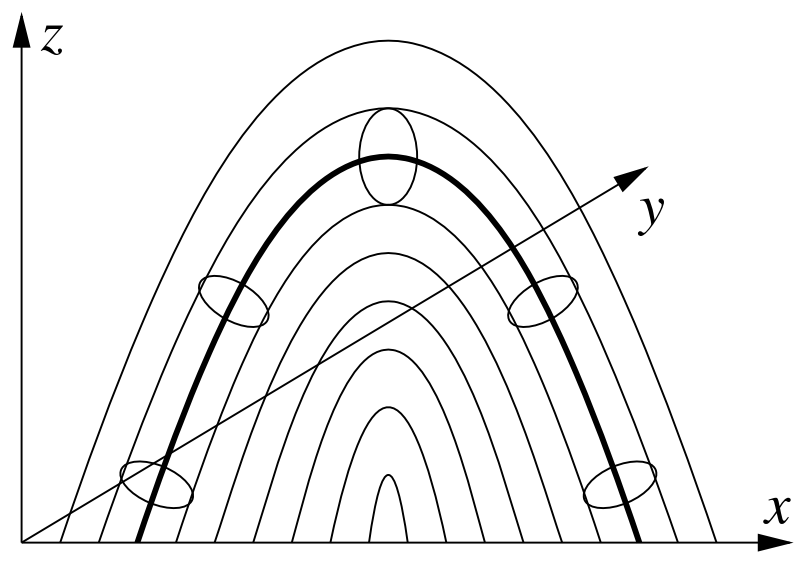

Fig. 1. The sketch of the equilibrium state. The ends of the magnetic loop are assumed to be frozen in a dense photospheric plasma. The axis of the magnetic loop is shown by the thick line. A few cross-sections of the loop are shown by ellipses.

where the loop is embedded in a two-dimensional potential magnetic field. The paper is organised as follows. In the next section we formulate the problem, describe the unperturbed state, and present the governing equations in curvilinear coordinates. In Sect. 3 we simplify the governing equations using the thin tube approximation. In Sect. 4 we study the eigenmodes of the vertical and horizontal loop oscillations in the approximation of weak tube expansion. In Sect. 5 we apply general results obtained in the previous sections to a particular equilibrium with exponentially decaying magnetic field. Section 6 presents the summary of the obtained results and our conclusions.

\section{Problem formulation and governing equations}

Let us introduce Cartesian coordinates $x, y, z$ with the $z$-axis in the vertical direction, and consider an equilibrium magnetic field $\boldsymbol{B}$ with the magnetic field lines in the $x z$-plane that is independent of $y$. Since the magnetic field is solenoidal, it follows that $\boldsymbol{B}$ can be expressed in terms of magnetic flux function $\psi$. We also assume that $\boldsymbol{B}$ is potential, so that it can be expressed in terms of potential $\phi$. As a result we have

$B_{x}=-B_{0} \frac{\partial \psi}{\partial z}=B_{0} \frac{\partial \phi}{\partial x}, \quad B_{z}=B_{0} \frac{\partial \psi}{\partial x}=B_{0} \frac{\partial \phi}{\partial z}$,

where $B_{0}$ is the characteristic value of the equilibrium magnetic field. We assume that the magnetic field is closed with the footpoints of the magnetic field lines frozen in the dense photospheric plasma. The sketch of a typical equilibrium state is shown in Fig. 1.

A magnetic field line is determined by the equations $\psi=$ const. and $y=$ const. The equation of the loop axis is $\psi=\psi_{0}$, where $\psi_{0}$ is a constant. We assume that this axis is in the $x z-$ plane. The equation of the loop boundary can be written in a parametric form as $\psi=\psi_{\mathrm{b}}(\eta), y=y_{\mathrm{b}}(\eta), \eta \in\left[\eta_{1}, \eta_{2}\right]$, where $\psi_{\mathrm{b}}\left(\eta_{1}\right)=\psi_{\mathrm{b}}\left(\eta_{2}\right)$ and $y_{\mathrm{b}}\left(\eta_{1}\right)=y_{\mathrm{b}}\left(\eta_{2}\right)$. The interior of the loop is defined by the equation

$\left(\psi-\psi_{0}\right)^{2}+y^{2}<\left[\psi(\eta)-\psi_{0}\right]^{2}+y^{2}(\eta)$,

while its exterior is defined by the equation

$\left(\psi-\psi_{0}\right)^{2}+y^{2}>\left[\psi(\eta)-\psi_{0}\right]^{2}+y^{2}(\eta)$.
We assume that the plasma density, $\rho$, is equal to $\rho_{\mathrm{i}}$ inside the loop, and $\rho_{\mathrm{e}}$ outside, where $\rho_{\mathrm{i}}$ and $\rho_{\mathrm{e}}$ are functions of $\phi$ only, and $\rho_{\mathrm{e}}<\rho_{i}$.

The plasma motion is described by linearised ideal MHD equations for cold plasmas,

$\rho \frac{\partial^{2} \boldsymbol{\xi}}{\partial t^{2}}=\frac{1}{\mu_{0}}(\nabla \times \boldsymbol{b}) \times \boldsymbol{B}$,

$\boldsymbol{b}=\nabla \times(\boldsymbol{\xi} \times \boldsymbol{B})$.

Here $\boldsymbol{\xi}$ is the plasma displacement, $\boldsymbol{b}$ the magnetic field perturbation, and $\mu_{0}$ the magnetic permeability of free space.

Now we introduce the curvilinear coordinates $\psi, y, \phi$. The unit vectors in this coordinate system are $\hat{\boldsymbol{\psi}}=\nabla \psi /|\nabla \psi|, \hat{\boldsymbol{y}}$ and $\hat{\boldsymbol{\phi}}=\nabla \phi /|\nabla \phi|$. It follows from Eq. (1) that $\hat{\boldsymbol{\phi}}=\boldsymbol{B} / B$, and the Cartesian components of $\hat{\psi}$ are given by

$\hat{\psi}=\frac{1}{B}\left(B_{z}, 0,-B_{x}\right)$.

It is straightforward to see that the introduced curvilinear coordinate system is orthogonal and right-oriented $(\hat{\boldsymbol{\phi}}=\hat{\boldsymbol{\psi}} \times \hat{\boldsymbol{y}})$. Since the coordinate system is orthogonal, the metric tensor is diagonal, and its diagonal elements are given by

$g_{\psi \psi}=g_{\phi \phi}=\frac{B_{0}^{2}}{B^{2}}, \quad g_{y y}=1$

Let us introduce the components of vectors $\boldsymbol{\xi}$ and $\boldsymbol{b}$,

$\boldsymbol{\xi}=\xi_{\psi} \hat{\boldsymbol{\psi}}+\xi_{y} \hat{\boldsymbol{y}}+\xi_{\phi} \hat{\boldsymbol{\phi}}, \quad \boldsymbol{b}=b_{\psi} \hat{\boldsymbol{\psi}}+b_{y} \hat{\boldsymbol{y}}+b_{\phi} \hat{\boldsymbol{\phi}}$

Then, using Eq. (5), the expression for the operator $\nabla \times$ in orthogonal curvilinear coordinates (e.g. Korn \& Korn 1961), and taking into account that, in accordance with (2), $\xi_{\psi}=0$, we rewrite Eqs. (2) and (3) in the coordinate form,

$\frac{\partial^{2} \xi_{\psi}}{\partial t^{2}}=\frac{B^{3}}{\mu_{0} \rho B_{0}}\left(\frac{\partial\left(b_{\psi} / B\right)}{\partial \phi}-\frac{\partial\left(b_{\phi} / B\right)}{\partial \psi}\right)$

$\frac{\partial^{2} \xi_{y}}{\partial t^{2}}=\frac{B^{2}}{\mu_{0} \rho B_{0}}\left(\frac{\partial b_{y}}{\partial \phi}-\frac{B_{0}}{B} \frac{\partial b_{\phi}}{\partial y}\right)$,

$b_{\psi}=\frac{B}{B_{0}} \frac{\partial\left(B \xi_{\psi}\right)}{\partial \phi}, \quad b_{y}=\frac{B^{2}}{B_{0}} \frac{\partial \xi_{y}}{\partial \phi}$,

$P=-\frac{B^{2}}{\mu_{0}}\left(\frac{1}{B_{0}} \frac{\partial\left(B \xi_{\psi}\right)}{\partial \psi}+\frac{\partial \xi_{y}}{\partial y}\right)$

where $P=\boldsymbol{B} \cdot \boldsymbol{b} / \mu_{0}=B b_{\phi} / \mu_{0}$ is the perturbation of the magnetic pressure. Eliminating $b_{\psi}$ and $b_{y}$ we rewrite Eqs. (6), (7) and (9) as

$\frac{\partial^{2} u}{\partial t^{2}}=-\frac{B^{4}}{\rho B_{0}} \frac{\partial Q}{\partial \psi}+\frac{B^{4}}{\mu_{0} \rho B_{0}^{2}} \frac{\partial^{2} u}{\partial \phi^{2}}$,

$\frac{\partial^{2} \xi_{y}}{\partial t^{2}}=-\frac{B^{2}}{\rho} \frac{\partial Q}{\partial y}+\frac{B^{2}}{\mu_{0} \rho B_{0}^{2}} \frac{\partial}{\partial \phi}\left(B^{2} \frac{\partial \xi_{y}}{\partial \phi}\right)$

$Q=-\frac{1}{\mu_{0}}\left(\frac{1}{B_{0}} \frac{\partial u}{\partial \psi}+\frac{\partial \xi_{y}}{\partial y}\right)$

where $u=B \xi_{\psi}$ and $Q=P / B^{2}$. Equations (10)-(12) constitute a closed system of equations for $u, \xi_{y}$ and $Q$. This system has to be 
supplemented by the boundary conditions at the tube boundary and footpoints. At the tube boundary the normal displacement and perturbation of the magnetic pressure have to be continuous. Since $B$ is continuous at the tube boundary, these conditions can be written as

$\left[\xi_{n}\right]=0, \quad[Q]=0$,

where $\xi_{n}$ is the component of displacement normal to the tube boundary, and the square brackets indicate the jump of a quantity across the boundary. The tube footpoints are determined by the equations $\phi=\phi_{1}$ and $\phi=\phi_{2}$. The magnetic field lines at the footpoints are frozen in the dense photospheric plasma, so that the displacement is equal to zero at the footpoints. This gives the boundary conditions

$u=0, \quad \xi_{y}=0 \quad$ at $\phi=\phi_{1,2}$.

The system of Eqs. (10)-(12) with the boundary conditions (13) and (14) is used in what follows to study the fast kink oscillations of curved coronal loops.

\section{Thin-tube approximation}

Let $L$ be the loop length and $a$ be the characteristic size of loop cross-sections. For typical coronal loops $a \ll L$, which inspires us to introduce $\epsilon=a / L \ll 1$. To have the same spatial scales of perturbation variation with respect to all three variables, $\psi, y$ and $\phi$, we introduce the new scaled variable $\varphi=\epsilon \phi$. Since the characteristic period of kink oscillations is equal to the transverse Alfvén time times $L / a$, we also introduce the scaled time $\tau=\epsilon t$. In new variables Eqs. (10)-(12) are rewritten as

$\frac{\partial^{2} u}{\partial \tau^{2}}=-\epsilon^{-2} \frac{B^{4}}{\rho B_{0}} \frac{\partial Q}{\partial \psi}+\frac{B^{4}}{\mu_{0} \rho B_{0}^{2}} \frac{\partial^{2} u}{\partial \varphi^{2}}$,

$\frac{\partial^{2} \xi_{y}}{\partial \tau^{2}}=-\epsilon^{-2} \frac{B^{2}}{\rho} \frac{\partial Q}{\partial y}+\frac{B^{2}}{\mu_{0} \rho B_{0}^{2}} \frac{\partial}{\partial \varphi}\left(B^{2} \frac{\partial \xi_{y}}{\partial \varphi}\right)$,

$Q=-\frac{1}{\mu_{0}}\left(\frac{1}{B_{0}} \frac{\partial u}{\partial \psi}+\frac{\partial \xi_{y}}{\partial y}\right)$

Now we look for solution to this system of equations in the form of expansions with respect to $\epsilon$. It follows from (15) and (16) that, if the expansions for $u$ and $\xi_{y}$ start from terms of the order of unity, then the expansion for $Q$ has to start from the term of the order of $\epsilon^{2}$. Hence, we write these expansions as

$u=u_{1}+\ldots, \quad \xi_{y}=\xi_{y 1}+\ldots, \quad Q=\epsilon^{2} Q_{1}+\ldots$,

where the dots indicate terms of higher order with respect to $\epsilon$. While the characteristic scale of variation of perturbations in the transverse directions is $a$, the characteristic scale of variation of equilibrium magnetic field in the transverse directions is $L$. This implies that, inside the loop and in its immediate vicinity we can write $B$ as

$B(\psi, \varphi)=B\left(\psi_{0}, \varphi\right)+O(\epsilon)$.

Substituting Eqs. (18) and (19) in Eqs. (15)-(17), and collecting terms of the lowest order with respect to $\epsilon$, we obtain

$\frac{\partial^{2} u_{1}}{\partial \tau^{2}}=-\frac{B^{4}}{\rho B_{0}} \frac{\partial Q_{1}}{\partial \psi}+\frac{B^{4}}{\mu_{0} \rho B_{0}^{2}} \frac{\partial^{2} u_{1}}{\partial \varphi^{2}}$, $\frac{\partial^{2} \xi_{y 1}}{\partial \tau^{2}}=-\frac{B^{2}}{\rho} \frac{\partial Q_{1}}{\partial y}+\frac{B^{2}}{\mu_{0} \rho B_{0}^{2}} \frac{\partial}{\partial \varphi}\left(B^{2} \frac{\partial \xi_{y 1}}{\partial \varphi}\right)$

$\frac{\partial u_{1}}{\partial \psi}+B_{0} \frac{\partial \xi_{y 1}}{\partial y}=0$

where $B$ is calculated at $\psi=\psi_{0}$. It follows from Eq. (22) that $u_{1}$ and $\xi_{y 1}$ can be expressed in terms of the flux function $\chi$,

$u_{1}=B_{0} \frac{\partial \chi}{\partial y}, \quad \xi_{y 1}=-\frac{\partial \chi}{\partial \psi}$.

Substituting these expressions in Eqs. (20) and (21), and using cross-differentiation to eliminate $Q_{1}$, we obtain the following equation for $\chi$,

$$
\begin{aligned}
\frac{\partial^{2}}{\partial \tau^{2}}\left(B^{2} \frac{\partial^{2} \chi}{\partial \psi^{2}}\right. & \left.+B_{0}^{2} \frac{\partial^{2} \chi}{\partial y^{2}}\right) \\
& -\frac{B^{4}}{\mu_{0} \rho B_{0}^{2}} \frac{\partial}{\partial \varphi}\left(B^{2} \frac{\partial^{3} \chi}{\partial \psi^{2} \partial \varphi}+B_{0}^{2} \frac{\partial^{3} \chi}{\partial y^{2} \partial \varphi}\right)=0 .
\end{aligned}
$$

Let us introduce the polar coordinates in the $\psi y$-plane,

$\psi=\psi_{0}+r \cos \theta, \quad y=r \sin \theta$

(recall that $\psi=\psi_{0}$ at the loop axis). The loop cross-section by the surface $\varphi=$ const. is, in general, a curved surface. Using the Taylor expansion we obtain

$$
\begin{aligned}
\varphi(x, z)-\varphi(\bar{x}, \bar{z})= & \frac{\partial \varphi}{\partial x}(x-\bar{x})+\frac{\partial \varphi}{\partial z}(z-\bar{z}) \\
& +O\left((x-\bar{x})^{2}+(z-\bar{z})^{2}\right)
\end{aligned}
$$

where $(\bar{x}, \bar{y}, \bar{z})$ is the point of intersection of the surface $\varphi=$ const. with the loop axis, and the partial derivatives on the righthand side of this expression are calculated at $x=\bar{x}$ and $z=\bar{z}$. The characteristic spatial scale of variation of function $\varphi(x, z)$ is $L$. This implies that the ratio of the last term on the right-hand side of this expression to the first two terms is of the order of $\epsilon$. Then it follows that, in the main order approximation with respect to $\epsilon$, the loop cross-section by the surface $\varphi=$ const. is a part of plane perpendicular to the loop axis. Cartesian coordinates in this plane are $y$ and $\sigma$. Since the $\sigma$-axis is orthogonal to $y$-axis and $\boldsymbol{B}$, we obtain that $\hat{\boldsymbol{\psi}}$ is the unit vector of the $\sigma$-axis.

In what follows we assume that the loop boundary is determined by the equation $r=a$. In the first order approximation with respect to $\epsilon$ the quantities $\sigma$ and $\psi$ are related by

$\sigma=\sqrt{g_{\psi \psi}}\left(\psi-\psi_{0}\right)=\left(B_{0} / B\right)\left(\psi-\psi_{0}\right)$.

Hence, once again in the first order approximation with respect to $\epsilon$, the equation of the loops boundary intersection with the $\sigma y$-plane is

$\sigma=a\left(B_{0} / B\right) \cos \theta, \quad y=a \cos \theta$.

This is the equation of an ellipse with the half-axes equal to $a$ and $a\left(B_{0} / B\right)$, where $B$ is calculated at the loop axis. We see that, while the axis parallel to the $y$-direction is constant, the perpendicular axis, in general, varies along the loop.

In what follows we are looking for eigenmodes of the loop oscillations and take perturbations of all quantities proportional to $\exp (-\mathrm{i} \Omega \tau)$. Then, in the new variables, Eq. (24) takes the form

$\frac{B^{4}}{\mu_{0} \rho B_{0}^{2}} \frac{\partial}{\partial \varphi} \mathcal{L}\left[\frac{\partial \chi}{\partial \varphi}\right]+\Omega^{2} \mathcal{L}[\chi]=0$, 
where

$$
\begin{aligned}
\mathcal{L}[\chi]= & \left(B^{2} \cos ^{2} \theta+B_{0}^{2} \sin ^{2} \theta\right) \frac{\partial^{2} \chi}{\partial r^{2}} \\
& +\left(B_{0}^{2} \cos ^{2} \theta+B^{2} \sin ^{2} \theta\right)\left(\frac{1}{r^{2}} \frac{\partial^{2} \chi}{\partial \theta^{2}}+\frac{1}{r} \frac{\partial \chi}{\partial r}\right) \\
& -\left(B^{2}-B_{0}^{2}\right)\left(\frac{1}{r} \frac{\partial^{2} \chi}{\partial r \partial \theta}-\frac{1}{r^{2}} \frac{\partial \chi}{\partial \theta}\right) \sin 2 \theta .
\end{aligned}
$$

The expression for $\mathcal{L}[\partial \chi / \partial \varphi]$ is obtained from the expression for $\mathcal{L}[\chi]$ by substituting $\partial \chi / \partial \varphi$ for $\chi$.

Let us now rewrite the boundary conditions (13) in terms of $\chi$. To transform the first boundary condition we need to calculate the normal vector to the loop boundary. Since $r=a$ at the boundary, and the Cartesian coordinates of a point are functions of $\psi$, $y$ and $\varphi$, the equation of the boundary in Cartesian coordinates can be written as

$$
\begin{aligned}
& x=x\left(\psi_{0}+a \cos \theta, \varphi\right), \\
& y=a \sin \theta, \\
& z=z\left(\psi_{0}+a \cos \theta, \varphi\right) .
\end{aligned}
$$

Here we have taken into account that $x$ and $z$ are functions of $\psi$ and $\varphi$, while they are independent of $y$. Equation (28) is the equation of the boundary written in a parametric form, $\theta$ and $\varphi$ being the parameters. The vectors

$\boldsymbol{l}_{\theta}=\left(\frac{\partial x}{\partial \theta}, \frac{\partial y}{\partial \theta}, \frac{\partial z}{\partial \theta}\right), \quad \boldsymbol{l}_{\varphi}=\left(\frac{\partial x}{\partial \varphi}, \frac{\partial y}{\partial \varphi}, \frac{\partial z}{\partial \varphi}\right)$,

are tangential to the boundary. To evaluate these vectors we use the relations

$$
\begin{array}{ll}
\frac{\partial x}{\partial \theta}=-a \sin \theta \frac{\partial x}{\partial \psi}, & \frac{\partial y}{\partial \theta}=a \cos \theta, \\
\frac{\partial z}{\partial \theta}=-a \sin \theta \frac{\partial z}{\partial \psi}, & \frac{\partial y}{\partial \varphi}=0 .
\end{array}
$$

We obtain with the aid of Eq. (1) that

$\left(\begin{array}{ll}\frac{\partial x}{\partial \psi} & \frac{\partial x}{\partial \phi} \\ \frac{\partial z}{\partial \psi} & \frac{\partial z}{\partial \phi}\end{array}\right)=\left(\begin{array}{ll}\frac{\partial \psi}{\partial x} & \frac{\partial \psi}{\partial z} \\ \frac{\partial \phi}{\partial x} & \frac{\partial \phi}{\partial z}\end{array}\right)^{-1}=\frac{B_{0}}{B^{2}}\left(\begin{array}{rr}B_{z} & B_{x} \\ -B_{x} & B_{z}\end{array}\right)$.

Using this result, Eq. (30), and the relation between $\varphi$ and $\phi$, yields

$\boldsymbol{l}_{\theta}=\frac{a}{B^{2}}\left(-B_{0} B_{z} \sin \theta, B^{2} \cos \theta, B_{0} B_{x} \sin \theta\right), \quad \boldsymbol{l}_{\varphi}=\frac{B_{0} \boldsymbol{B}}{\epsilon B^{2}}$.

The unit normal vector to the boundary is then given by

$$
\begin{aligned}
\boldsymbol{n}=\frac{\boldsymbol{l}_{\varphi} \times \boldsymbol{l}_{\theta}}{\left|\boldsymbol{l}_{\theta} \times \boldsymbol{l}_{\varphi}\right|} & =N\left(B_{z} \cos \theta, B_{0} \sin \theta,-B_{x} \cos \theta\right) \\
& =N\left(B \hat{\boldsymbol{\psi}} \cos \theta+B_{0} \hat{\boldsymbol{y}} \sin \theta\right),
\end{aligned}
$$

where

$N=\left(B^{2} \cos ^{2} \theta+B_{0}^{2} \sin ^{2} \theta\right)^{-1 / 2}$.

When deriving this expression we have used Eq. (4). The normal component of the displacement is given by

$$
\begin{aligned}
\xi_{n}=\boldsymbol{n} \cdot \boldsymbol{\xi} & =N\left(u \cos \theta+B_{0} \xi_{y} \sin \theta\right) \\
& =N B_{0}\left(\frac{\partial \chi}{\partial y} \cos \theta-\frac{\partial \chi}{\partial \psi} \sin \theta\right)=\frac{N B_{0}}{r} \frac{\partial \chi}{\partial \theta} .
\end{aligned}
$$

This expression is only valid in the lowest order approximation with respect to $\epsilon$ because, when deriving it, we substituted $u_{1}$ and $\xi_{y 1}$ for $u$ and $\xi_{y}$. Using the expression for $\xi_{n}$, and taking into account that $B$ and, consequently, $N$ is continuous at the loop boundary, we reduce the first boundary condition in (13) to

$$
[\chi]=0 \text { at } r=a .
$$

To rewrite the second boundary condition in (13) in terms of $\chi$ we need to express $Q_{1}$ in terms of $\chi$. To do this we first take $u_{1}, \xi_{y 1}$ and $Q_{1}$ proportional to $\exp (-\mathrm{i} \Omega \tau)$. Then we transform Eqs. (20) and (21) to the variables $r$ and $\theta$, multiply the first equation by $\sin \theta$, the second by $B^{2} \sin \theta$, and add the results. As a result, with the aid of (23), we obtain the expression for $\partial Q_{1} / \partial \theta$ in terms of $\chi$. Substituting this expression in the second boundary condition in (13), that has been preliminarily differentiated with respect to $\theta$, we arrive at

$\left[\frac{B^{4}}{\mu_{0} B_{0}^{2}} \frac{\partial}{\partial \varphi} \mathcal{M}\left[\frac{\partial \chi}{\partial \varphi}\right]+\rho \Omega^{2} \mathcal{M}[\chi]\right]=0 \quad$ at $\quad r=a$,

where

$\mathcal{M}[\chi]=\left(B^{2} \cos ^{2} \theta+B_{0}^{2} \sin ^{2} \theta\right) \frac{\partial \chi}{\partial r}-\frac{\left(B^{2}-B_{0}^{2}\right)}{2 a} \frac{\partial \chi}{\partial \theta} \sin 2 \theta$,

and the expression for $\mathcal{M}[\partial \chi / \partial \varphi]$ is obtained from the expression for $\mathcal{M}[\chi]$ by substituting $\partial \chi / \partial \varphi$ for $\chi$.

Finally, it follows from (14) and (23) that $\chi$ is constant at $\varphi=$ $\varphi_{1,2}$. Since $\chi$ is determined with the accuracy up to an additive function of $\varphi$, we can take

$\chi=0 \quad$ at $\varphi=\varphi_{1,2}$.

\section{Eigenmodes of weakly expanding loops}

To make analytical progress we consider weakly expanding loops and assume that the variation of the magnetic field magnitude along the loop is small. In accordance with this assumption we take

$B^{2}=B_{0}^{2}[1+\lambda q(\varphi)]$

and assume that $\lambda \ll 1$, while the function $q(\varphi)$ is of the order of unity. Then we use the regular perturbation method and look for the solution in the form

$\chi=\chi_{1}+\lambda \chi_{2}, \quad \Omega=\Omega_{1}+\lambda \Omega_{2}$.

\subsection{First order approximation}

In the first order approximation we substitute the expansions (36) and (37) in Eq. (26), and boundary conditions (32), (33) and (35). As a result we obtain

$\left(\frac{B_{0}^{2}}{\mu_{0} \rho} \frac{\partial^{2}}{\partial \varphi^{2}}+\Omega_{1}^{2}\right)\left(\frac{\partial^{2} \chi_{1}}{\partial r^{2}}+\frac{1}{r} \frac{\partial \chi_{1}}{\partial r}+\frac{1}{r^{2}} \frac{\partial^{2} \chi_{1}}{\partial \theta^{2}}\right)=0$

$\left[\chi_{1}\right]=\left[\left(\frac{B_{0}^{2}}{\mu_{0}} \frac{\partial^{2}}{\partial \varphi^{2}}+\rho \Omega_{1}^{2}\right) \frac{\partial \chi_{1}}{\partial r}\right]=0$ at $r=a$,

$\chi_{1}=0 \quad$ at $\quad \varphi=\varphi_{1,2}$. 
If we denote the expression in the second brackets in Eq. (38) as $f$, then it follows from Eq. (40) that $f=0$ at $\varphi=\varphi_{1,2}$. As a result we obtain the Sturm-Liouville problem for the function $f$,

$\frac{B_{0}^{2}}{\mu_{0} \rho} \frac{\partial^{2} f}{\partial \varphi^{2}}+\Omega_{1}^{2} f=0, \quad f=0 \quad$ at $\quad \varphi=\varphi_{1,2}$.

Since $B_{0}^{2} /\left(\mu_{0} \rho\right)$ is the square of the Alfvén speed, this SturmLiouville problem describes Alfvénic oscillations (inside the loop when $\rho=\rho_{\mathrm{i}}$, and outside when $\rho=\rho_{\mathrm{e}}$ ). In what follows we assume that the frequencies of the loop kink oscillations do not coincide with the Alfvénic frequencies either inside or outside the loop. Then $\Omega_{1}^{2}$ is not an eigenvalue of the Sturm-Liouville problem (41), and the only solution of this problem is $f=0$. This implies that Eq. (38) reduces to

$\frac{\partial^{2} \chi_{1}}{\partial r^{2}}+\frac{1}{r} \frac{\partial \chi_{1}}{\partial r}+\frac{1}{r^{2}} \frac{\partial^{2} \chi_{1}}{\partial \theta^{2}}=0$

In what follows we only study the kink oscillations, so that we take

$\chi_{1}=\chi_{\mathrm{c}}(r, \varphi) \cos \theta+\chi_{\mathrm{s}}(r, \varphi) \sin \theta$.

Substituting this expression in Eq. (42) we obtain

$\frac{\partial^{2} \chi_{\mathrm{c}, \mathrm{s}}}{\partial r^{2}}+\frac{1}{r} \frac{\partial \chi_{\mathrm{c}, \mathrm{s}}}{\partial r}-\frac{\chi_{\mathrm{c}, \mathrm{s}}}{r^{2}}=0$.

In addition, the functions $\chi_{\mathrm{c}}(r, \varphi)$ and $\chi_{\mathrm{s}}(r, \varphi)$ have to satisfy the boundary conditions (39). The solution to Eq. (44) regular at $r=0$, vanishing as $r \rightarrow \infty$, and satisfying the first boundary condition in (39), is

$\chi_{\mathrm{c}, \mathrm{s}}(r, \varphi)=A_{\mathrm{c}, \mathrm{s}}(\varphi) \begin{cases}r, & r<a, \\ a^{2} / r, & r>a .\end{cases}$

Substituting this solution in the second boundary condition in (39) yields

$\frac{\mathrm{d}^{2} A_{\mathrm{c}, \mathrm{s}}}{\mathrm{d} \varphi^{2}}+\frac{\Omega_{1}^{2}}{C_{k}^{2}} A_{\mathrm{c}, \mathrm{s}}=0, \quad C_{k}^{2}=\frac{2 B_{0}^{2}}{\mu_{0}\left(\rho_{\mathrm{i}}+\rho_{\mathrm{e}}\right)}$.

This equation coincides with the equation derived by Dymova \& Ruderman (2005) for kink oscillations of a straight loop with the constant circular cross-section. This is not surprising at all because, in the first order approximation with respect to $\lambda$, we neglected the effect of variation of the cross-section along the loop. It follows from (40) that the functions $A_{\mathrm{c}}(\varphi)$ and $A_{\mathrm{s}}(\varphi)$ have to satisfy the boundary conditions

$A_{\mathrm{c}, \mathrm{s}}(\varphi)=0 \quad$ at $\quad \varphi=\varphi_{1,2}$.

Equation (46) and the boundary condition (47) constitute the Sturm-Liouville problem. This problem has a non-trivial solution only when $\Omega_{1}^{2}$ is an eigenvalue. In that case the solution is unique with the accuracy up to multiplication by an arbitrary constant. Let us denote this solution as $A_{1}(\varphi)$. Then

$A_{\mathrm{c}}(\varphi)=C_{\mathrm{c}} A_{1}(\varphi), \quad A_{\mathrm{s}}(\varphi)=C_{\mathrm{s}} A_{1}(\varphi)$

where $C_{\mathrm{c}}$ and $C_{\mathrm{s}}$ are (in general complex) constants.

\subsection{Second order approximation}

In the second order approximation we collect terms proportional to $\lambda$ in Eq. (26), and boundary conditions (32), (33) and (35). Then, using (43), (45), (46) and (48), we obtain

$\frac{\partial^{2} \chi_{2}}{\partial r^{2}}+\frac{1}{r} \frac{\partial \chi_{2}}{\partial r}+\frac{1}{r^{2}} \frac{\partial^{2} \chi_{2}}{\partial \theta^{2}}=0, \quad r<a$,

$$
\begin{aligned}
\left(\frac{B_{0}^{2}}{\mu_{0} \rho_{\mathrm{e}}} \frac{\partial^{2}}{\partial \varphi^{2}}\right. & \left.+\Omega_{1}^{2}\right)\left(\frac{\partial^{2} \chi_{2}}{\partial r^{2}}+\frac{1}{r} \frac{\partial \chi_{2}}{\partial r}+\frac{1}{r^{2}} \frac{\partial^{2} \chi_{2}}{\partial \theta^{2}}\right)= \\
& -\frac{2}{r^{3}}\left(\frac{B_{0}^{2}}{\mu_{0} \rho_{\mathrm{e}}} \frac{\partial}{\partial \varphi} q \frac{\partial A_{1}}{\partial \varphi}+q \Omega_{1}^{2} A_{1}\right) \\
& \times\left(C_{\mathrm{c}} \cos 3 \theta+C_{\mathrm{s}} \sin 3 \theta\right), \quad r>a,
\end{aligned}
$$$$
\left[\chi_{2}\right]=0 \quad \text { at } \quad r=a \text {, }
$$

$\left[\left(\frac{B_{0}^{2}}{\mu_{0}} \frac{\partial^{2}}{\partial \varphi^{2}}+\rho \Omega_{1}^{2}\right) \frac{\partial \chi_{2}}{\partial r}\right]=\frac{B_{0}^{2}}{2 \mu_{0}} \frac{\mathrm{d}}{\mathrm{d} \varphi} q \frac{\mathrm{d} A_{1}}{\mathrm{~d} \varphi}$

$$
\begin{aligned}
& \times\left(3 C_{\mathrm{c}} \cos \theta+C_{\mathrm{s}} \sin \theta+C_{\mathrm{c}} \cos 3 \theta+C_{\mathrm{s}} \sin 3 \theta\right) \\
&- \frac{q}{2} A_{1} \Omega_{1}^{2}\left[2 \rho_{\mathrm{i}}\left(C_{\mathrm{c}} \cos \theta+2 C_{\mathrm{s}} \sin \theta\right)\right. \\
&+\left.\rho_{\mathrm{e}}\left(3 C_{\mathrm{c}} \cos \theta+3 C_{\mathrm{s}} \sin \theta-C_{\mathrm{c}} \cos 3 \theta-C_{\mathrm{s}} \sin 3 \theta\right)\right] \\
&+ 2 \Omega_{1} \Omega_{2} A_{1}\left(\rho_{\mathrm{i}}+\rho_{\mathrm{e}}\right)\left(C_{\mathrm{c}} \cos \theta+C_{\mathrm{s}} \sin \theta\right) \quad \text { at } \quad r=a, \\
& \chi_{2}=0 \quad \text { at } \varphi=\varphi_{1,2} .
\end{aligned}
$$

Equation (49) for $\chi_{2}$ inside the loop coincides with Eq. (42) for $\chi_{1}$. It can be easily verified that $\chi=r\left[F_{\mathrm{c}}(\varphi) \cos \theta+F_{\mathrm{s}}(\varphi) \sin \theta\right]$, where $F_{\mathrm{c}}(\varphi)$ and $F_{\mathrm{s}}(\varphi)$ are arbitrary functions, is an exact solution of Eq. (26). This property is related to the fact that, in the thin tube approximation, the loop moves as rigid, i.e. the displacement of all points of a particular cross-section are the same. The solution to Eq. (49) is

$\left.\chi_{2}=r\left[A_{2 \mathrm{c}}(\varphi) \cos \theta+A_{2 \mathrm{c}}(\varphi) \sin \theta\right)\right], \quad r<a$,

where $A_{2 \mathrm{c}}(\varphi)$ and $A_{2 \mathrm{e}}(\varphi)$ are arbitrary functions. It follows from (53) that $A_{2 \mathrm{c}, \mathrm{s}}\left(\varphi_{1,2}\right)=0$.

We look for the solution to Eq. (50) in the form

$\chi_{2}=r^{-1}\left[\widetilde{A}_{2 \mathrm{c}}(\varphi) \cos \theta+{\widetilde{A_{2 s}}}_{(\varphi) \sin \theta]}\right.$

$$
+\tilde{\chi}_{2}(r, \varphi)\left(C_{\mathrm{c}} \cos 3 \theta+C_{\mathrm{s}} \sin 3 \theta\right) .
$$

Substituting this expression in Eq. (50) we obtain

$$
\begin{aligned}
\left(\frac{B_{0}^{2}}{\mu_{0} \rho_{\mathrm{e}}} \frac{\partial^{2}}{\partial \varphi^{2}}\right. & \left.+\Omega_{1}^{2}\right)\left(\frac{\partial^{2} \tilde{\chi}_{2}}{\partial r^{2}}+\frac{1}{r} \frac{\partial \tilde{\chi}_{2}}{\partial r}-\frac{9}{r^{2}} \tilde{\chi}_{2}\right)= \\
& -\frac{2}{r^{3}}\left(\frac{B_{0}^{2}}{\mu_{0} \rho_{\mathrm{e}}} \frac{\partial}{\partial \varphi} q \frac{\partial A_{1}}{\partial \varphi}+q \Omega_{1}^{2} A_{1}\right) .
\end{aligned}
$$

The general solution to this equation is given by

$\tilde{\chi}_{2}=a^{2} r^{-1} A_{3}(\varphi)+r^{-3} f(\varphi)$,

where $f(\varphi)$ is an arbitrary function, and $A_{3}(\varphi)$ satisfies the equation

$\frac{B_{0}^{2}}{\mu_{0} \rho_{\mathrm{e}}} \frac{\mathrm{d}^{2} A_{3}}{\mathrm{~d} \varphi^{2}}+\Omega_{1}^{2} A_{3}=\frac{B_{0}^{2}}{4 \mu_{0} \rho_{\mathrm{e}}} \frac{\mathrm{d}}{\mathrm{d} \varphi} q \frac{\mathrm{d} A_{1}}{\mathrm{~d} \varphi}+\frac{q}{4} \Omega_{1}^{2} A_{1}$. 
Substituting (55) in (51) and using (57) we obtain $f(\varphi)=$ $-a^{4} A_{3}(\varphi), \widetilde{A}_{2 \mathrm{c}}=a^{2} A_{2 \mathrm{c}}(\varphi)$, and $\widetilde{A}_{2 \mathrm{c}}=a^{2} A_{2 \mathrm{c}}(\varphi)$, so that eventually

$$
\begin{aligned}
\chi_{2}= & \frac{a^{2}}{r}\left[A_{2 \mathrm{c}}(\varphi) \cos \theta+A_{2 \mathrm{~s}}(\varphi) \sin \theta\right) \\
& +A_{3}(\varphi)\left(\frac{a^{2}}{r}-\frac{a^{4}}{r^{3}}\right)\left(C_{\mathrm{c}} \cos 3 \theta+C_{\mathrm{s}} \sin 3 \theta\right), \quad r>a .
\end{aligned}
$$

It follows from (53) that $A_{3}\left(\varphi_{1,2}\right)=0$.

Now we substitute (54) and (59) in the boundary condition (52), and collect terms proportional to $\cos \theta, \sin \theta$, and $\left(C_{\mathrm{c}} \cos 3 \theta+C_{\mathrm{s}} \sin 3 \theta\right)$. As a result we obtain equations for $A_{2 \mathrm{c}}$, $A_{2 s}$, and $A_{3}$. The equation for $A_{3}$ coincides with (58), and the equations for $A_{2 c}$ and $A_{2 s}$ are

$$
\begin{aligned}
\frac{\mathrm{d}^{2} A_{2 \mathrm{c}}}{\mathrm{d} \varphi^{2}}+\frac{\Omega_{1}^{2}}{C_{k}^{2}} A_{2 \mathrm{c}}= & -C_{\mathrm{c}}\left(\frac{3}{4} \frac{\mathrm{d}}{\mathrm{d} \varphi} q \frac{\mathrm{d} A_{1}}{\mathrm{~d} \varphi}\right. \\
& \left.-\frac{q \mu_{0}}{4 B_{0}^{2}}\left(2 \rho_{\mathrm{i}}+3 \rho_{\mathrm{e}}\right) \Omega_{1}^{2} A_{1}+\frac{2 \Omega_{1} \Omega_{2}}{C_{k}^{2}} A_{1}\right), \\
\frac{\mathrm{d}^{2} A_{2 \mathrm{~s}}}{\mathrm{~d} \varphi^{2}}+\frac{\Omega_{1}^{2}}{C_{k}^{2}} A_{2 \mathrm{~s}}= & -C_{\mathrm{s}}\left(\frac{1}{4} \frac{\mathrm{d}}{\mathrm{d} \varphi} q \frac{\mathrm{d} A_{1}}{\mathrm{~d} \varphi}\right. \\
& \left.-\frac{q \mu_{0}}{4 B_{0}^{2}}\left(4 \rho_{\mathrm{i}}+3 \rho_{\mathrm{e}}\right) \Omega_{1}^{2} A_{1}+\frac{2 \Omega_{1} \Omega_{2}}{C_{k}^{2}} A_{1}\right) .
\end{aligned}
$$

The homogeneous counterparts to Eqs. (60) and (61) coincide with Eq. (49), so that they have a non-trivial solution $A_{1}(\varphi)$. Then it follows from the general theory of linear operators that Eqs. (60) and (61) can be solved only when their righthand sides are orthogonal to $A_{1}(\varphi)$. These solvability conditions can be also obtained directly. To do this we multiply either of Eqs. (60) and (61) by $A_{1}$ and integrate with respect to $\varphi$ from $\varphi_{1}$ to $\varphi_{2}$. Using integration by parts and the boundary conditions $A_{2 \mathrm{c}, \mathrm{s}}\left(\varphi_{1,2}\right)=0$ we obtain that the integrals from the left-hand sides of Eqs. (60) and (61) are equal to zero. This implies that the integrals from the right-hand sides of Eqs. (60) and (61) are also equal to zero. Once again using integration by parts we can write these conditions as

$$
\begin{aligned}
& C_{\mathrm{c}}\left(\Omega_{2}-\Omega_{2 \mathrm{~h}}\right)=0, \quad \Omega_{2 \mathrm{~h}} \int_{\varphi_{1}}^{\varphi_{2}} \frac{A_{1}^{2}}{C_{k}^{2}} \mathrm{~d} \varphi=\int_{\varphi_{1}}^{\varphi_{2}} \frac{q}{8}\left[\frac { \mu _ { 0 } \Omega _ { 1 } } { B _ { 0 } ^ { 2 } } \left(2 \rho_{\mathrm{i}}\right.\right. \\
& \left.\left.+3 \rho_{\mathrm{e}}\right) A_{1}^{2}+\frac{3}{\Omega_{1}}\left(\frac{\mathrm{d} A_{1}}{\mathrm{~d} \varphi}\right)^{2}\right] \mathrm{d} \varphi, \\
& C_{\mathrm{s}}\left(\Omega_{2}-\Omega_{2 \mathrm{v}}\right)=0, \quad \Omega_{2 \mathrm{v}} \int_{\varphi_{1}}^{\varphi_{2}} \frac{A_{1}^{2}}{C_{k}^{2}} \mathrm{~d} \varphi=\int_{\varphi_{1}}^{\varphi_{2}} \frac{q}{8}\left[\frac { \mu _ { 0 } \Omega _ { 1 } } { B _ { 0 } ^ { 2 } } \left(4 \rho_{\mathrm{i}}\right.\right. \\
& \left.\left.+3 \rho_{\mathrm{e}}\right) A_{1}^{2}+\frac{1}{\Omega_{1}}\left(\frac{\mathrm{d} A_{1}}{\mathrm{~d} \varphi}\right)^{2}\right] \mathrm{d} \varphi .
\end{aligned}
$$

In general, $\Omega_{2 h} \neq \Omega_{2 v}$. Then it follows from (60) and (61) that either $C_{\mathrm{c}} \neq 0$ and $\Omega_{2}=\Omega_{2 h}$, or $C_{\mathrm{s}} \neq 0$ and $\Omega_{2}=\Omega_{2 v}$. Using (23), (25), (43), (45), (48), and the relation $u=B \xi_{\psi}$, we easily obtain

$\xi_{\psi 1}=C_{\mathrm{s}} A_{1}(\varphi), \quad \xi_{y 1}=-C_{\mathrm{c}} A_{1}(\varphi)$.

This result implies that the case where $C_{\mathrm{s}} \neq 0$ corresponds to vertical oscillations, and the case where $C_{\mathrm{c}} \neq 0$ to horizontal oscillations. We see that the second order approximation removes the system degeneration. Now eigenmodes cannot be polarised in any direction. Rather they are polarised either in the vertical or in horizontal direction.

\subsection{Equations in physical variables}

In the first order approximation with respect to $\lambda$ the eigenfrequencies and eigenmodes of the loop kink oscillations are determined by Eq. (46) with the boundary conditions (47). The second order corrections to the eigenfrequencies are given by Eqs. (62) and (63). All these equations are written in variables that are inconvenient for comparison with the results obtained in previous studies and with observations. We now rewrite these equations in physical variables. We start from introducing the length $s$ along the loop axis. Since $g_{\phi \phi}=\left(B_{0} / B\right)^{2}$, we obtain $\mathrm{d} s=\left(B_{0} / B\right) \mathrm{d} \phi=\epsilon^{-1}\left(B_{0} / B\right) \mathrm{d} \varphi$, so that

$$
s=B_{0} \int_{\phi_{1}}^{\phi} \frac{\mathrm{d} \phi^{\prime}}{B\left(\phi^{\prime}\right)}=\epsilon^{-1} B_{0} \int_{\varphi_{1}}^{\varphi} \frac{\mathrm{d} \varphi^{\prime}}{B\left(\varphi^{\prime}\right)} .
$$

We also introduce $\xi_{\mathrm{v}}=\epsilon \xi_{\psi 1}, \xi_{\mathrm{h}}=\epsilon \xi_{y 1}$, and $\tilde{\omega}_{1}=\epsilon^{-1} \Omega_{1}$. In these new variables Eq. (46) with the boundary conditions (47) takes the form

$\frac{1}{B} \frac{\mathrm{d}}{\mathrm{d} s}\left(\frac{1}{B} \frac{\mathrm{d} \tilde{\xi}}{\mathrm{d} s}\right)+\frac{\tilde{\omega}_{1}^{2}}{B_{0}^{2} C_{k}^{2}} \tilde{\xi}=0, \quad \tilde{\xi}=0 \quad$ at $\quad s=0, L$

where $L$ is the length of the loop given by

$L=B_{0} \int_{\phi_{1}}^{\phi_{2}} \frac{\mathrm{d} \phi}{B(\phi)}$

and either $\tilde{\xi}=\xi_{\mathrm{v}}$, or $\tilde{\xi}=\xi_{\mathrm{h}}$. Now we recall that $B^{2}=$ $B_{0}^{2}[1+\lambda q(s)]$ and look for the solution to the boundary value problem (66) in the form $\tilde{\xi}=\xi+\lambda \xi_{2}$ and $\tilde{\omega}_{1}=\omega_{1}+\lambda \tilde{\omega}_{2}$. Then we obtain in the first order approximation with respect to $\lambda$

$\frac{\mathrm{d}^{2} \xi}{\mathrm{d} s^{2}}+\frac{\omega_{1}^{2}}{C_{k}^{2}} \xi=0, \quad \xi=0 \quad$ at $\quad s=0, L$,

where either $\xi=\xi_{\mathrm{v}}+O(\lambda)$, or $\xi=\xi_{\mathrm{h}}+O(\lambda)$. The boundary value problem (68) coincides with one obtained by Dymova \& Ruderman (2005) for kink oscillations of a straight non-expanding loop with the circular cross-section and with the density varying along the loop.

In the next order approximation we obtain

$\frac{\mathrm{d}^{2} \xi_{2}}{\mathrm{~d} s^{2}}+\frac{\omega_{1}^{2}}{C_{k}^{2}} \xi_{2}=\frac{\lambda}{2}\left(\frac{\mathrm{d}}{\mathrm{d} s} q \frac{\mathrm{d} \xi}{\mathrm{d} s}-\frac{\omega_{1}^{2}}{C_{k}^{2}} q \xi-\frac{4 \omega_{1} \tilde{\omega}_{2}}{C_{k}^{2}} \xi\right)$,

with $\xi_{2}=0$ at $s=0, L$. Multiplying this equation by $q$, integrating the result with respect to $s$, and using (68) and integration by parts, we obtain the solvability condition for this boundary value problem,

$\tilde{\omega}_{2} \int_{0}^{L} \frac{\xi^{2}}{C_{k}^{2}} \mathrm{~d} \xi=-\frac{1}{4 \omega_{1}^{2}} \int_{0}^{L} q\left[\left(\frac{\mathrm{d} \xi}{\mathrm{d} s}\right)^{2}+\frac{\omega_{1}^{2}}{C_{k}^{2}} \xi^{2}\right] \mathrm{d} s$.

Now we introduce $\omega=\epsilon^{-1} \Omega$ and write $\omega=\omega_{1}+\lambda \omega_{2}$. Then we have

$\omega_{1}+\lambda \omega_{2}=\epsilon^{-1}\left(\Omega_{1}+\lambda \Omega_{2}\right)=\tilde{\omega}_{1}+\lambda \epsilon^{-1} \Omega_{2}$,

so that $\omega_{2}=\epsilon^{-1} \Omega_{2}+\tilde{\omega}_{2}$. Using this result, Eq. (70), and Eqs. (62) and (63) rewritten in terms of $s$ and $\xi$, we eventually obtain expressions for the frequency of vertical, $\omega_{\mathrm{v}}$, and horizontal, $\omega_{\mathrm{h}}$, oscillations of the loop,

$\omega_{\mathrm{v}, \mathrm{h}}=\omega_{1}+\lambda \omega_{2 \mathrm{v}, \mathrm{h}}$ 
where

$\omega_{2 \mathrm{v}} \int_{0}^{L} \frac{\xi^{2}}{C_{k}^{2}} \mathrm{~d} s=\int_{0}^{L} \frac{q}{8}\left[\frac{\mu_{0} \omega_{1}}{B_{0}^{2}}\left(3 \rho_{\mathrm{i}}+2 \rho_{\mathrm{e}}\right) \xi^{2}-\frac{1}{\omega_{1}}\left(\frac{\mathrm{d} \xi}{\mathrm{d} s}\right)^{2}\right] \mathrm{d} s$,

$\omega_{2 \mathrm{~h}} \int_{0}^{L} \frac{\xi^{2}}{C_{k}^{2}} \mathrm{~d} s=\int_{0}^{L} \frac{q}{8}\left[\frac{\mu_{0} \omega_{1}}{B_{0}^{2}}\left(\rho_{\mathrm{i}}+2 \rho_{\mathrm{e}}\right) \xi^{2}+\frac{1}{\omega_{1}}\left(\frac{\mathrm{d} \xi}{\mathrm{d} s}\right)^{2}\right] \mathrm{d} s$.

To verify the correctness of the obtained results we consider kink oscillations of a straight homogeneous magnetic tube with an elliptic cross-section. To do this we take $q$ to be a non-zero constant. Then the half-axes of the elliptic cross-section are $a$ and $b=a(1-\lambda q / 2)$. The solution to the boundary value problem (68) corresponding to the fundamental mode is $\xi=\sin (\pi s / L), \omega_{1}=$ $\pi C_{k} / L$. Calculation of $\omega_{2 \mathrm{v}}$ and $\omega_{2 \mathrm{~h}}$ is straightforward, and eventually we obtain

$\omega_{2 \mathrm{v}}=\frac{\pi q C_{k}\left(5 \rho_{\mathrm{i}}+3 \rho_{\mathrm{e}}\right)}{8 L\left(\rho_{\mathrm{i}}+\rho_{\mathrm{e}}\right)}, \quad \omega_{2 \mathrm{~h}}=\frac{\pi q C_{k}\left(3 \rho_{\mathrm{i}}+5 \rho_{\mathrm{e}}\right)}{8 L\left(\rho_{\mathrm{i}}+\rho_{\mathrm{e}}\right)}$.

Kink oscillations of a straight homogeneous magnetic tube with an elliptic cross-section were studied by Ruderman (2003). In particular, he obtained the expressions for the frequencies of two kink modes in the thin tube approximation (see his Eq. (60)). If we substitute $b=a(1-\lambda q / 2)$ and $B^{2}=B_{0}^{2}(1+\lambda q)$ in these expressions, and make expansions with respect to $\lambda$ up to terms proportional to $\lambda$, then we obtain exactly the same expressions for $\omega_{2 \mathrm{v}}$ and $\omega_{2 \mathrm{~h}}$.

\section{Exponentially decaying magnetic field in an isothermal atmosphere}

To give an example of the obtained general results we consider the following equilibrium state. The potential and flux function of the equilibrium magnetic field are give by

$\phi=l e^{-k z} \sin (k x), \quad \psi=l e^{-k z} \cos (k x)$.

The picture of the field lines for this magnetic field is qualitatively the same as one shown in Fig. 1. The ratio of densities inside and outside the loop is constant, $\rho_{\mathrm{i}} / \rho_{\mathrm{e}}=\zeta>1$. The loop is in the isothermal atmosphere, so that

$\rho_{\mathrm{e}}=\rho_{0} \mathrm{e}^{-z / H}$,

where $H$ is the atmospheric scale height. The loop footpoints are at $z=0, x= \pm x_{0}$, so that $\psi_{0}=l \cos \left(k x_{0}\right)$. Then the equation of the loop axis is

$\mathrm{e}^{k z}=\frac{\cos (k x)}{\cos \left(k x_{0}\right)}, \quad|k x| \leq k x_{0}$.

Using (74) and (76) we can express $z$ in terms of $\phi$,

$\mathrm{e}^{-2 k z}=l^{-2} \phi^{2}+\cos ^{2}\left(k x_{0}\right)$.

It follows from (1) and (74) that

$B_{x}=B_{0} l k \mathrm{e}^{-k z} \cos (k x), \quad B_{z}=-B_{0} l k \mathrm{e}^{-k z} \sin (k x)$,

so that

$\frac{B^{2}}{B_{0}^{2}}=1+\lambda q(\phi)=(l k)^{2} \mathrm{e}^{-2 k z}=k^{2} \phi^{2}+(l k)^{2} \cos ^{2}\left(k x_{0}\right)$.
This equation implies that

$\lambda q(\phi)=k^{2} \phi^{2}+(l k)^{2} \cos ^{2}\left(k x_{0}\right)-1$.

The function $|\phi(x, z)|$ varies from $l \sin \left(k x_{0}\right)$ at the footpoints to zero at the loop apex, so that $\lambda q(\phi)$ varies from $(l k)^{2}-1$ to $(l k)^{2} \cos ^{2}\left(k x_{0}\right)-1$. The condition $|q(\phi)| \lesssim 1$ implies that

$(l k)^{2}=1+O(\lambda), \quad \cos ^{2}\left(k x_{0}\right)=1+O(\lambda)$.

It follows from the second equation in (81) that $\left(k x_{0}\right)^{2}=O(\lambda)$. Since $\lambda$ is an arbitrary small parameter, we will take

$\lambda=\left(k x_{0}\right)^{2}$

in what follows. To satisfy the first Eq. in (81) we take

$l=k^{-1}(1+\alpha \lambda)$

where $\alpha$ is a constant of the order of unity. Then it follows from (80) that

$q(\phi)=x_{0}^{-2} \phi^{2}+2 \alpha-1+O(\lambda)$.

Now we can rewrite the equation of the loop axis (76) in an approximate form,

$z=\frac{1}{2} x_{0} \sqrt{\lambda}\left[1-\left(\frac{x}{x_{0}}\right)^{2}\right]$

We see that the loop shape is approximately parabolic. The ratio of the loop height to the distance between the loop footpoints is $\frac{1}{4} \sqrt{\lambda}$, so that it is small.

The loop cross-section is elliptic with the horizontal halfaxis equal to $a$. The vertical half-axis is equal to $\left(B_{0} / B\right) a \approx$ $a\left(1-\frac{1}{2} \lambda q\right)$. Hence, in accordance with (74) and (82)-(84), it monotonically increases with the height from $a(1-\alpha \lambda)$ at the loop footpoints to $a[1-(\alpha-1 / 2) \lambda]$ at the loop apex.

The distance along the loop is related to the magnetic field potential by Eq. (65). Using (74), (79) and (84) we obtain from this equation

$$
\begin{aligned}
s & \approx \int_{-x_{0}[1+(\alpha-1 / 6) \lambda]}^{\phi}\left[1-\frac{\lambda}{2}\left(x_{0}^{-2} \phi^{\prime 2}+2 \alpha-1\right)\right] \mathrm{d} \phi^{\prime} \\
& =\phi+x_{0}+\lambda\left[\left(\alpha-\frac{1}{6}\right) x_{0}-\frac{\phi^{3}+x_{0}^{3}}{6 x_{0}^{2}}-\left(\alpha-\frac{1}{2}\right)\left(\phi+x_{0}\right)\right] .
\end{aligned}
$$

Substituting $\phi=x_{0}[1+(\alpha-1 / 6) \lambda]$ in (85) we obtain the expression for the loop length, $L \approx(2+\lambda / 3) x_{0}$. Using (85) we rewrite the expression for $q$ as

$q(s)=\left(s / x_{0}-1\right)^{2}+2 \alpha-1+O(\lambda)$.

Now we assume that $H$ and $L$ are of the same order of magnitude. Since the loop height is of the order of $L \sqrt{\lambda}$, this implies that the relative density variation along the loop axis is of the order of $\sqrt{\lambda}$. The account of this density variation would result in a correction to $\omega_{1}$ of the order of $\sqrt{\lambda}$. This correction is the same for the vertical and horizontal oscillations. Since we are mainly interested in the difference of frequencies of the vertical and horizontal oscillations, we will neglect the density variation in what follows. Then $C_{k}=$ const. and the solution to the boundary value problem (68) corresponding to the fundamental mode is

$\xi=\xi_{0} \sin \frac{\pi s}{L}, \quad \omega_{1}=\frac{\pi C_{k}}{L}$, 
where $\xi_{0}$ is a constant. The calculation of integrals in (72) and (73) with $q(s)$ given by (86) and $\xi$ given by (87) is straightforward, and we obtain

$\omega_{2 \mathrm{v}}=\frac{C_{k}\left[\pi^{2}(5 \zeta+3)(6 \alpha+1)-6(7 \zeta+5)\right]}{24 \pi^{2} L(\zeta+1)}$,

$\omega_{2 \mathrm{~h}}=\frac{C_{k}\left[\pi^{2}(3 \zeta+5)(6 \alpha+1)-6(\zeta+3)\right]}{24 \pi^{2} L(\zeta+1)}$.

When deriving these expressions we have taken $x_{0} \approx L / 2$. The difference between the frequencies of vertical and horizontal oscillations is

$$
\begin{aligned}
\Delta \omega & =\omega_{\mathrm{v}}-\omega_{\mathrm{h}}=\lambda\left(\omega_{2 \mathrm{v}}-\omega_{2 \mathrm{~h}}\right) \\
& =\frac{\lambda C_{k}\left[\pi^{2}(\zeta-1)(6 \alpha+1)-6(3 \zeta+1)\right]}{12 \pi^{2} L(\zeta+1)} .
\end{aligned}
$$

We see that, in general, $\omega_{\mathrm{v}} \neq \omega_{\mathrm{h}}$. They are only equal when

$\alpha=\frac{3 \zeta+1}{\pi^{2}(\zeta-1)}-\frac{1}{6}$.

It is especially instructive to take $\alpha=0$. In that case the loop cross-sections at the footpoints are circles of radius $a$. The vertical half-axis $b$ of the elliptic loop cross-section monotonically increases with the height from $a$ at the loop footpoints to $a(1+\lambda / 2)$ at the loop apex. Substituting $\alpha=0$ in (90) we obtain

$\Delta \omega=-\frac{\lambda C_{k}(3 \zeta+1)}{2 \pi^{2} L(\zeta+1)}$,

so that $\omega_{\mathrm{v}}<\omega_{\mathrm{h}}$. This result is in good agreement with the results obtained by Ruderman (2003). Ruderman (2003) has shown that the frequency of kink oscillation of a magnetic tube with an elliptic cross section in the direction of the larger axis is smaller than that in the direction of the smaller axis. Since the vertical axis of the variable elliptic loop cross-section is everywhere larger than the horizontal axis, we should expect that the frequency of the vertical oscillation is smaller than that of the horizontal oscillation.

\section{Summary and conclusions}

In this paper we have studied kink oscillations of curved coronal magnetic loops with the density varying along the loop. We have assumed that the loop is in a vertical plane, so that the loop torsion is zero. We have also assumed that the equilibrium magnetic field is potential. Using the magnetic field potential and flux function as curvilinear coordinates in the loop plane we derived the equation governing the loop motion in the thin tube approximation. Then we have considered a loop with the elliptic cross-section, and rewrote the governing equations and the boundary conditions at the loop boundary in terms of polar coordinates in the loop cross-section. An important property of this model is that the loop curvature causes the loop expansion. As a result the ratio of axis of the loop cross-section varies along the loop.

The governing equation for the loop motion is a partial differential equation for one function that depends of the polar coordinates in the loop cross-section, $r$ and $\theta$. The coefficients of this equation depend both on $r$ and $\theta$. We managed to find the solution to the governing equation corresponding to kink oscillations inside the loop. However, it seems highly improbable that a similar solution describing the motion outside the loop can be found. To make analytical progress we assumed that the loop expansion is small and introduced the small parameter, $\lambda$, characterising this expansion. In this approximation the loop crosssection is almost circular. The ratio of axes of the loop elliptic cross-section differs from unity by a quantity of the order of $\lambda$. After that we have used the regular perturbation method and looked for the solution to the problem in the form of power series expansions with respect to $\lambda$.

In particular, we looked for the eigenmodes of kink oscillations in the form $\omega=\omega_{1}+\lambda \omega_{2}$. In the first order approximation we found that $\omega_{1}$ is determined by the eigenvalue problem (68). Equation (68) is exactly the same as one obtained by Dymova \& Ruderman (2005) for kink oscillations of a straight non-expanding loop with the circular cross-section and the density varying along the loop. The eigenvalue problem is degenerate in the sense that kink oscillations can be polarised in any direction.

In the next order approximation the kink eigenmodes of the loop oscillation can be polarised only either in the vertical or horizontal direction, so that the degeneration of the eigenvalue problem is removed. The corrections to the kink oscillation frequency are different for the vertical and horizontal oscillations. They are given by Eqs. (72) and (73) for the fundamental modes of the vertical and horizontal kink oscillations.

As an example we have considered a simple equilibrium with the magnetic field magnitude exponentially decaying with the height. Neglecting the density variation along the loop we reduced the eigenvalue problem (68) to one describing kink oscillations of a straight thin homogeneous magnetic tube. Then we easily calculated the corrections to the frequencies of the vertical and horizontal oscillations, $\omega_{\mathrm{v}}$ and $\omega_{\mathrm{h}}$ (see Eqs. (88) and (89)). These corrections depend on the parameter $\alpha$ characterising the shape of the loop cross-section at the footpoints. In general, this cross-section is an ellipse with the ration of the vertical and horizontal axes equal to $1-\alpha \lambda$. When $\alpha=0$, which corresponds to the circular footpoint cross-section, $\omega_{\mathrm{v}}<\omega_{\mathrm{h}}$, i.e. the frequency of the vertical loop oscillation is smaller than that of the horizontal oscillation.

The main conclusion of this work is that the frequencies of the vertical and horizontal kink oscillations are, in general, different. The effect of loop curvature on the frequencies of the vertical and horizontal kink oscillations is not direct. Rather the curvature results in variation of the shape of the loop crosssection along the loop, and it is this variation of the cross-section shape that causes the difference in the frequencies. It is instructive to compare the results of this work with those obtained by Van Doorsselaere et al. (2004) and Terradas et al. (2006). These authors came to a conclusion that the difference between the frequencies of the vertical and horizontal oscillations of a curved loop is only of the order of $\epsilon^{2}$, where $\epsilon$ is the ration of the loop radius to its length, so that it can be neglect in the thin tube approximation. Our study clearly shows that this result in related to the model used by Van Doorsselaere et al. (2004) and Terradas et al. (2006). In this model a curved loop has a circular cross-section with the constant radius.

The difference between the frequencies of the vertical and horizontal oscillations is of the order of $\lambda \ll 1$, i.e. it is small. This result is directly related to our assumption that the loop expansion is small. For loops with sufficiently large expansions this difference can be quite substantial. 
Although, as we have already mentioned, the majority of observed kink oscillations of coronal loops are horizontally polarised, an arbitrary disturbance should cause both vertically and horizontally polarised oscillations. Since, in general, the frequencies of the vertical and horizontal oscillations are different, it would be interesting to look for signatures of two different frequencies in the observational data. If the ratio of two observed frequencies is sufficiently large (say, larger than 1.5), then they are usually attributed to the fundamental mode and first overtone of kink oscillations. However if two frequencies with a smaller ratio (say, 1.2 or 1.3) are found, then it is quite probable that they are the frequencies of the vertical and horizontal kink oscillations.

Acknowledgements. The author acknowledges support by an STFC grant.

\section{References}

Aschwanden, M. J., Fletcher, L., Schrijver, C. J., \& Alexander, D. 1999, ApJ, 520,880

Dymova, M., \& Ruderman, M. S. 2005, Sol. Phys., 229, 79

Korn, G. A., \& Korn, T. M. 1961, Mathematical handbook for scientists and engineers (New York: McGraw-Hill)

Nakariakov, V. M., Ofman, L., DeLuca, E. E., Roberts, B., \& Davila, J. M. 1999, Science, 285, 862

Ruderman, M. S. 2003, A\&A, 409, 287

Ruderman, M. S., \& Erdélyi, R., Space. Sci. Rev., in press

Terradas, J., Oliver, R., \& Ballester, J. L. 2006, ApJ, 650, L91

Van Doorsselaere, T., Debosscher, A., Andries, J., \& Poedts, S. 2004, A\&A, 424, 1065

Wang, T. J., \& Solanki, S. K. 2004, A\&A, 421, L33

Wang, T. J., Solanki, S. K., \& Selwa, M. 2008, A\&A, 489, 1307 